\title{
ЭКСПЛОРАТОРНЫЙ ФАКТОРНЫЙ АНАЛИЗ ДЛЯ ВЫЯВЛЕНИЯ КЛЮЧЕВЫХ ВНУТРИФИРМЕННЫХ ФАКТОРОВ ИЗ ФИНАНСОВОЙ ОТЧЕТНОСТИ ФУТБОЛЬНЫХ КЛУБОВ"
}

\author{
(C) 2020 Карлик Александр Евсеевич \\ доктор экономических наук, профессор, заведующий кафедрой экономики \\ и управления предприятиями и производственными комплексами \\ Санкт-Петербургский государственный экономический университет, Россия, Санкт-Петербург \\ E-mail: karlik1@mail.ru

\section{(c) 2020 Платонов Владимир Владимирович} \\ доктор экономических наук, профессор, \\ профессор кафедры экономики и управления предприятиями и производственными комплексами \\ Санкт-Петербургский государственный экономический университет, Россия, Санкт-Петербург \\ E-mail: vladimir.platonov@gmail.com
}

\section{(c) 2020 Кузяев Далер Адьямович}

аспирант кафедры экономики и управления предприятиями и производственными комплексами Санкт-Петербургский государственный экономический университет, Россия, Санкт-Петербург

$$
\text { E-mail: daler9593@mail.ru }
$$

Предложен и апробирован когнитивный подход к анализу информации финансовой отчетности для получения более обоснованных результатов, требующихся для реализации новых методов управления, ориентированных на нематериальное производство. Подход реализуется путем эксплораторного факторного анализа для выявления ключевых внутрифирменных факторов и апробируется на финансовой отчетности футбольных клубов. Результаты анализа показали достаточно высокую степень надежности финансовой информации для применения когнитивного подхода для нематериальных отраслей и реального сектора.

Ключевые слова: когнитивная экономика; финансовая отчетность; организационные инновации; новые методы управления; футбол

Введение. В информационно-сетевой экономике, высокая эффективность все в большей степени обеспечивается внедрением новых технологий и нематериальными факторами. Для предприятий нематериальных отраслей применение традиционного финансового анализа часто оказывается недостаточно информативным, так как он узко направлен на выявление того, как результат соотносится с ресурсами - задействованными (активы и пассивы) и израсходованными. Такой подход применим в случае преобладания материальных факторов, но недостаточно информативен для предприятий нематериальных отраслей и предприятий реального сектора, где высока доля интеллектуального капитала [2]. Научные исследования по стратегическому анализу и, в особенности, в рамках ресурсно- ориентированного подхода показали, что, наряду с ресурсами, эффективность предприятий, где велика доля нематериальных факторов, является результатом системного взаимодействия технологий, индивидуальных компетенций и ряда других ключевых факторов, таких как динамические способности [1]. Обширная информация финансовой отчетности, составленная в соответствии с Международными стандартами финансовой отчетности (МСФО) и национальными правилами бухучета разных стран, используется для традиционного финансового анализа, которому присуще описанное выше ограничение. Многие предприятия нематериальных отраслей обеспечивают не только экономический, но и социальный эффект, например, индустрия спорта. Для проведения активной экономической

\footnotetext{
* Исследование выполнено при финансовой поддержке РФФИ в рамках научного проекта № 18-010-00971 «Исследование новых форм межфирменного взаимодействия и организации в реальном секторе в условиях информационно-сетевой экономики».
} 
политики, внедрения новых методов управления в этих отраслях, требуется формирование инструментария финансового анализа с использованием когнитивных технологий, основывающихся на резко возросших возможностях технологий цифровой экономики. В данной статье, мы обосновываем решение указанной задачи путем применения эксплораторного факторного анализа.

Обоснование когнитивного подхода к анализу финансовой отчетности на основе эксплораторного факторного анализа. Привычный финансовый анализ показывает различия в финансовой эффективности между предприятиями, а факторы, их обуславливающие, остаются скрытыми от аналитика. В рамках традиционного подхода, уникальные внутрифирменные факторы - ресурсы и организационные способности отражает экономическая добавленная стоимость (EVA) или эффект от использования интеллектуального капитала [2], но эти факторы все равно скрыты за статьями финансовой отчетности, непосредственно учитывающими только движение ресурсов и результатов. Иными словами, то, что предприятие А имеет более высокую эффективность, чем предприятие Б, хорошая новость для менеджмента и инвесторов, но плохая - для науки: это различие показывает, что неучтенными, скрытыми от анализа оказались ключевые внутрифирменные факторы, обуславливающие данное различие.

Привычный детерминированный факторный анализ основывается на предпосылке наличия функциональной связи между факторами экономическими индикаторами и обобщающим показателем. Таким образом, связь между обобщающим показателем и определяющими его факторами определяется функциями элементарной алгебры. Длительное время такой простой инструмент позволял решать прикладные задачи. Проблема возникла с распространением современных технологически-интенсивных предприятий и ростом нематериальных отраслей. Такие предприятия и организации представляют сложную хозяйственную систему, состоящую из множества взаимосвязанных разнородных элементов, причем наличие сетевого взаимодействия между элементами означает неоднозначность причинно-следственных зависимостей. Применение стохастического факторного анализа к отраслям нематериального производства и инновационно-активным предприятиям реального сектора дает более реали- стичные результаты, но принципиально проблему не решает. Он не может идентифицировать факторы, обуславливающие различия в эффективности сложных хозяйственных систем, ни при дедуктивном, ни при индуктивном подходе, так как одной из предпосылок его применения является качественная однородность по совокупности изучаемых связей.

Существующий подход к учету и анализу информации констатирует различия в эффективности между предприятиями из-за того, что при использовании одного и того же количества материальных ресурсов получен разный эффект. Необходим подход, который показывал бы одновременно различия в эффекте и соответствующие им различия в ключевых внутрифирменных факторах: не только в ресурсах, но и в организационных способностях, обусловивших наблюдаемую разницу в эффекте. В идеале, создание такого подхода, обеспечивающего как учет, так и анализ ключевых внутрифирменных факторов, требует формирование новой системы учета, а это - отдаленная перспектива. Следовательно, задача состоит в том, чтобы использовать имеющуюся финансовую отчетность, чтобы вскрыть скрытые за данными финансового учета внутрифирменные факторы. Необходимо найти подходы к ее решению, без принципиальных изменений существующей системы бухгалтерского учета, так как это очень дорого и займет десятилетия. Альтернативный вариант - когнитивный подход. Он состоит в выявлении причин, глубинных факторов, стоящих за изменчивостью статей финансовой отчётности через когнитивное восприятие этих факторов бухгалтерами, при подготовке финансовой отчетности.

В строгом понимании любая финансовая отчетность является не объективным, а субъективным феноменом, когнитивным процессом, учитывающим установленные стандарты и правила. Иными словами, первичная информация проходит через ментальное сито [7]. Эксплораторный факторный анализ представляет строгий научный метод изучения когнитивного, ментального процесса. Он позволяет получить объективные оценки субъективных суждений. При новом подходе, показатели финансовой отчетности компаний рассматриваются как такие субъективные суждения, хотя и выполненные по принятым стандартам и правилам. Эксплораторный факторный анализ позволяет выявить латентные, скрытые за показателями финансо- 
вой отчетности объективные факторы экономики предприятия. Это -объективная оценка ресурсов, искаженных при субъективной оценке, и организационных способностей, которые никак не показаны в официальной отчетности.

Согласно исследованиям в области управления сложными системами, модель финансовой отчетности построена одновременно на: 1) классической логике, которая позволяет уменьшить неопределенность сложной хозяйственной системы (сократить комплексность); 2) немонотонной логике, для которой выполняются не все законы классической логики [5]. Прежде всего, речь идет о законе исключения третьего. Согласно ему, из двух высказываний «А» или «не А» одно - обязательно истинно. Так, если есть два суждения, одно из которых формулирует отрицание другого, то они не могут быть одновременно ложными. Это позволяет сократить комплексность - неопределенность сложной хозяйственной системы путем обобщения и абстракции, но для этого требуется человеческое суждение. Надо не сетовать на субъективизм, свойственный финансовой отчетности/ и тешить себя иллюзиями, что от него избавятся с повсеместным переходом к МСФО. Следует признать, что без субъективных суждений невозможно учесть комплексность реального бизнеса и дать такое суждение - задача бухгалтера. Эксплораторный факторный анализ извлекает объективную информацию из этих суждений. Таким образом, содержанием когнитивного подхода к финансовому анализу является выявление ключевых факторов экономики предприятия на основе профессионального суждения бухгалтеров и аудиторов, подготовивших финансовую отчетность.

Объект исследования. Объектом исследования, в данной статье, являются профессиональные футбольные клубы, финансовые данные которых взяты из базы данных финансовой отчетности (отчет о финансовом положении и отчета о прибылях и убытках) по европейским публичным и частным компаниям Amadeus версии 2019919 [3]. Размер выборки был определен, исходя из трех критериев: 1) вида деятельности - профессиональный футбол; 2) объема выручки; 3) актуальности отчетности; 4). качества финансовой отчетности. В случае наличия консолидированной и неконсолидированной отчетности, для одного клуба, приоритет отдавался последней. В первоначальную выборку вошли 128 футбольных клубов с выручкой боль- ше 12 млн. евро: 41 из Англии; 19 из Испании; 15 из Италии, 13 из Франции; 10 из Германии; по 6 из Бельгии и Шотландии; по 4 из России и Португалии; по 2 из Уэльса и Дании, по 1 из Австрии, Болгарии, Греции, Турции, Украины и Чехии. Как и следовало ожидать, большинство команд представляют пять «больших» лиг Европы (74,6\%). Высокая доля клубов Великобритании (32\%) отражает, как популярность и финансовую силу клубов британских профессиональных лиг, так и большее развитие финансовой отчетности для этого вида деятельности. Российские клубы оказались представлены лучше, чем ряд наших соседей по таблице клубных коэффициентов УЕФА.

Дальнейший отбор осуществлялся, исходя из критериев актуальности и качества информации. По 76 клубам - отчетность за 2019, по 43 клубам - за 2018, по 4 клубам - за 2017 год. В соответствии с критерием качества отчетности, были отсортированы клубы, в финансовой отчетности которых отсутствовала информация по важным статьям. Так как предметом исследования являлись внутрифирменные факторы, определяемые внутренней средой компании, а не внешние факторы, зависящие от рыночной конъюнктуры, в случае возникновения дилеммы качество - актуальность финансовой отчетности, приоритет отдавался качеству. В итоге, в соответствии с указанными выше критериями, в выборку для эксплораторного факторного анализа вошло 107 клубов. Крупнейшие клубы, по объему ресурсов, в целом совпадают с ведущими клубами Европы (Таблица 1). Футбольные клубы, в выборке, отличаются очень высокой долей нематериальных активов - больше трети. Средний клуб в выборке имеет 253,13 млн. евро. активов, в том числе 163,81 млн. руб. основных средств, и среднюю операционную выручку равную 160,61 млн. евро. По структуре активов выборка, включающая клубы разного размера, достаточно однородна.

Переменные модели. Так как при эксплораторном анализе скрытых факторов, переменные не подразделяются на зависимые и независимые, был задан набор исходных переменных, представляющий собой статьи финансовой отчетности. В состав переменных включались статьи активов баланса (отчета о финансовом положении) и статьи отчета о прибылях и убытках, в части эффекта деятельности. Для анализа использовалось 10 переменных, отражающих объемы задействованных ресурсов (активы, ос- 
Таблица 1. Значения основных ресурсных переменных для 12 крупнейших футбольных клубов по объему активов (2019 год, млн. евро)

\begin{tabular}{|l|c|c|c|c|c|c|c|c|c|}
\hline \multicolumn{1}{|c|}{ Футбольный клуб } & Активы & \multicolumn{2}{|c|}{$\begin{array}{c}\text { Основной } \\
\text { капитал }\end{array}$} & $\begin{array}{c}\text { Нематериальные } \\
\text { активы }\end{array}$ & \multicolumn{2}{|c|}{$\begin{array}{c}\text { Основные } \\
\text { средства }\end{array}$} & \multicolumn{2}{|c|}{$\begin{array}{c}\text { Оборотный } \\
\text { капитал }\end{array}$} \\
\hline Манчестер Юнайтед & 1383 & 583 & $42 \%$ & 380 & $27 \%$ & 185 & $13 \%$ & 800 & $58 \%$ \\
\hline Манчестер Сити (*) & 1360 & 1014 & $75 \%$ & 551 & $41 \%$ & 463 & $34 \%$ & 346 & $25 \%$ \\
\hline Барселона & 1359 & 956 & $70 \%$ & 542 & $40 \%$ & 206 & $15 \%$ & 403 & $30 \%$ \\
\hline Реал Мадрид & 1138 & 726 & $64 \%$ & 315 & $28 \%$ & 350 & $31 \%$ & 413 & $36 \%$ \\
\hline Париж Сен-Жермен (*) & 1076 & 636 & $59 \%$ & 452 & $42 \%$ & 94 & $9 \%$ & 440 & $41 \%$ \\
\hline Арсенал & 1040 & 756 & $73 \%$ & 271 & $26 \%$ & 478 & $46 \%$ & 284 & $27 \%$ \\
\hline Атлетико Мадрид & 980 & 683 & $70 \%$ & 234 & $24 \%$ & 383 & $39 \%$ & 298 & $30 \%$ \\
\hline Ливерпуль & 840 & 635 & $76 \%$ & 418 & $50 \%$ & 217 & $26 \%$ & 205 & $24 \%$ \\
\hline Интернационале & 830 & 477 & $58 \%$ & 441 & $53 \%$ & 21 & $3 \%$ & 352 & $42 \%$ \\
\hline Ювентус (*) & 773 & 548 & $71 \%$ & 366 & $47 \%$ & 162 & $21 \%$ & 224 & $29 \%$ \\
\hline Бавария (*) & 737 & 448 & $61 \%$ & 175 & $24 \%$ & 258 & $35 \%$ & 289 & $39 \%$ \\
\hline Челси & 709 & 693 & $98 \%$ & 531 & $75 \%$ & 161 & $23 \%$ & 16 & $2 \%$ \\
\hline Итого по 12 клубам & 11516 & 7464 & $65 \%$ & 4147 & $36 \%$ & 2818 & $24 \%$ & 4052 & $35 \%$ \\
\hline $\begin{array}{l}\text { По остальным 95 } \\
\text { клубам }\end{array}$ & 15570 & 10064 & $65 \%$ & 6248 & $40 \%$ & 2851 & $18 \%$ & 5505 & $35 \%$ \\
\hline
\end{tabular}

Источник [3].

(*) Данные за 2018 год

новные средства, нематериальные активы, дебиторская задолженность, прочие оборотные активы), и переменные эффекта (операционная выручка, финансовые доходы, финансовые расходы, сальдо движения денежных средств от операционный деятельности, чистая прибыль).

Согласно научным методическим рекомендациям [11], при определении минимально допустимого размера выборки, мы руководствовались тремя критериями. Первый критерий - абсолютное количество наблюдений, которое должно быть не меньше 100, так называемое «Правило 100» [8]. Второй критерий состоит в том, что число наблюдений должно превышать количество переменных более, чем в 5 раз [10]. Согласно третьему критерию, если размер выборки близок к нижнему порогу (100), то количество переменных должно не превышать $20[9,4]$.

Оценка надежности когнитивного подхода к анализу финансовой отчетности на основе эксплораторного факторного анализа. При определении надежности анализировались 10 исходных переменных. Расчеты осуществлены в программе IBM SPSS Statistics. Версия 23. Количественные оценки переменных, в 107 наблюдениях в представленной выборке, рассматривались как результат профессионального суждения аудиторов и бухгалтеров о реальных внутрифирменных факторах, руководствовавшихся стандартами финансовой отчетности. Оценка надежности финансовой отчетности рассматривалась как согласованность субъективных суждений между собой. Каждое наблюдение в выборке рассматривается как тест, исследующий набор конструктов - исходные переменные финансовой отчетности, отражающие субъективные оценки - профессиональные суждения (Таблица 2). Важнейшим количественным критерием надежности является показатель внутренней согласованности - коэффициент Альфа Кронбаха (Таблица 3).

Полученное значение коэффициента Альфа Кронбаха следует рассматривать как хорошее [6], что свидетельствует о согласованности между собой данных из разных наблюдений (разных клубов), бухгалтера которых количественно измеряли одни и те же 10 конструктов.

Методические указания по реализации когнитивного подхода к анализу финансовой отчетности на основе эксплораторного факторного анализа. В отличие от регрессионного анализа, метод эксплораторного анализа латентных факторов не предполагает предварительного разделения на зависимые и независимые переменные. Эти преимущества делают метод уникальным для проведения поискового исследования, позволяя обработать первичные 
Таблица 2. Сводный отчет по наблюдениям

\begin{tabular}{|c|c|c|}
\hline Наблюдения & $\mathrm{N}$ & $\%$ \\
\hline Валидные & 107 & 100,0 \\
\hline Исключено & 0 & 0 \\
\hline Всего & 107 & 100,0 \\
\hline
\end{tabular}

Таблица 3. Статистика надежности

\begin{tabular}{|c|c|}
\hline Альфа Кронбаха & N элементов \\
\hline 0,831 & 10 \\
\hline
\end{tabular}

данные с минимальной субъективной компонентой, способствуя объективному, независящему от заранее сформировавшихся установок исследователей по изучению внутренней структуры фирмы как сложной хозяйственной системы. Применение анализа скрытых факторов позволяет сократить большое количество статей финансовой отчетности до меньшего количества существенных переменных, которые должны стать предметом более глубокого анализа и содержательной интерпретации. Это - важное преимущество для преодоления сложности изучаемого объекта (комплексности) при обосновании стратегических решений. Оно обусловлено особенностью эксплораторного факторного анализа, заключающейся в том, что известные переменные объясняются меньшим количеством неизвестных переменных и случайной ошибки. Эта особенность позволяет сократить большое количество исходных переменных (статей отчетности) до меньшего количества компонент, которые затем идентифицируются как внутрифирменные факторы и им дается содержательная интерпретация. Тем самым, выявляется реальная структура изучаемого объекта. Из двух методов - эксплораторного и конфирматорного (подтверждающего) факторного анализа, предпочтение должно быть отдано первому методу, так как цель рассматриваемого здесь исследования состоит в обеспечении процесса принятия решения, а не в подтверждении заранее заданной теории. Смысл когнитивного подхода к анализу финансовой отчетности, на основе эксплораторного факторного анализа, заключается в том, чтобы объективно оценить субъективное профессиональное суждение составителей отчетности и обосновать применяемые решения.

Для расчетов при эксплораторном анализе латентных факторов, целесообразно выбрать метод главных компонент, при котором максимальная вариация приписывается первому фактору, а после ее исключения, второй фактор объясняется следующей по размеру вариацией и так далее. Тем самым, достигается перераспределение дисперсии между компонентами и получается максимально простая и наглядная структура факторов. Именно такая структура необходима для использования результатов анализа в управленческой процедуре. На основе количественных оценок и содержания статей финансовой отчетности, в наибольшей степени нагружающих ту или иною компоненту, можно составить суждение о характере латентных факторов.

Заключение. Результаты проведенного исследования показали надежность использования информации финансовой отчетности для эксплораторного факторного анализа. Таким образом, этот подход к конкретным исследованиям, может рассматриваться как перспективный вариант для обеспечения новых методов управления, для которых требуется учет ключевых внутрифирменных факторов эффективности и результативности. Вместе с тем, это - только первый шаг к апробированию данного метода. После оценки надежности, следующим шагом к применению данного метода в исследованиях по экономике фирмы является оценка валидности, то есть допустимости эксплораторного факторного анализа для обоснования управленческих решений. Иными словами, необходимо удостовериться, что предлагаемый метод измеряет ключевые факторы экономики фирмы, а не что-то другое. Этот момент важен для определения целесообразности усилий по внедрению эксплораторного факторного анализа информации бухгалтерского учета компаний реального сектора и нематериальных отраслей. 


\section{Библиографический список}

1. Карлик А.Е., Платонов В.В. Ресурсно-ориентированный подход в междисциплинарной перспективе // Финансы и бизнес, 2013. - № 4. С. 4-12.

2. Платонов В.В. Финансовые аспекты оценки интеллектуального капитала // Финансы и бизнес.- 2006.№ $1 .-$ C. $98-111$.

3. Amadeus database.-Brussels: Bureau van Dijk - 2020.- [Электронный ресурс] (https://amadeus.bvdinfo.com/ version-2019919).

4. Arrindell, W. A., van der Ende. J. An Empirical Test of the Utility of the Observations to Variables Ratio in Factor and Components Analysis // Applied Psychological Measurement.-1985.- No 9.- pp.165-178.

5. Banerjee $S$. Chaos and Complexity Theory for Management: Nonlinear Dynamics. - Hershey: IGI Global, 2012, 449 pages.

6. DeVellis R.F. Scale development: Theory and applications. Thousand Oaks: Sage Publications 2003. 184 pages.

7. Bergman J.-P., Knutas A., Jantunen A., Tarkiainen A., Luukka P., Karlik A., Platonov V. Strategic Interpretation on Sustainability Issues: Eliciting Cognitive Maps of Boards of Directors. Corporate Governance // The International Journal of Business in Society. - 2016. - Vol.1.- No.16.- pp. 162-186.

8. Gorsuch R.L. Factor analysis. Toronto: Saunders. 1983. 359 pages.

9. Gorsuch R. L. Factor analysis (2nd ed.). Hillsdale NJ: Lawrence Erlbaum Associates. 1983. 425 pages.

10. Hatcher L. A Step-by-Step Approach to Using the SAS System for Factor Analysis and Structural Equation Modeling/ The SAS Institute. Review. Cary, NC - 1994. pp. 325-339.

11. MacCallum R.C., Widaman K. F., Zhang S., Hong S. Sample size in factor analysis // Psychological Methods. - 1999.No 4.- pp. 84-99. 